\title{
Tropism between hepatic and pulmonary metastases in colorectal cancers
}

\author{
SUNG-HYUN KIM ${ }^{1 *}$, SO-JUNG CHOI ${ }^{1 *}$, JOON SUK PARK ${ }^{2}$, JINSEON LEE $^{1}$, YONG BEOM CHO $^{3}$, \\ MIN-WOONG KANG ${ }^{5}$, WOO YONG LEE ${ }^{3}$, YONG SOO $\mathrm{CHOI}^{2}$, HONG KWAN KIM ${ }^{2}$, \\ JOUNGHO HAN $^{4}$, HO-KYUNG CHUN ${ }^{3}$ and JHINGOOK KIM ${ }^{1,2}$ \\ ${ }^{1}$ Samsung Biomedical Research Institute, Departments of ${ }^{2}$ Thoracic Surgery, ${ }^{3}$ Surgery and ${ }^{4}$ Pathology, Samsung Medical \\ Center, Sungkyunkwan University, School of Medicine, Seoul 135-710; ${ }^{5}$ Department of Thoracic and Cardiovascular \\ Surgery, Chungnam National University, Daejeon 305-764, Chungnam, Republic of Korea
}

Received February 10, 2012; Accepted April 23, 2012

DOI: $10.3892 /$ or.2012.1837

\begin{abstract}
In metastatic colorectal cancers, tumor cells are disseminated prior to surgical resection of the primary tumor but remain dormant until proper colonization mechanisms are activated. To identify the colonization mechanisms of the metastatic tumors, we conducted a pairwise comparison between primary colorectal cancers and metastatic tumors ( $n=12$ pairs), including six hepatic pairs and six pulmonary pairs. The mRNA levels of 224 genes previously reported to be associated with metastasis, cytokines and angiogenesis were quantitatively determined by PCR arrays. Among them, 27 genes were duplicated or triplicated to show consistent expression. Unsupervised hierarchical clustering of the $\mathrm{Ct}$ values of metastasis-related genes revealed that liver metastases were indistinguishable from primary colorectal cancers $(n=5 / 6)$, whereas lung metastases were highly diversified from one another and from the primary tumors $(n=6 / 6)$. Cytokines and receptor gene expression array data also confirmed the divergence of pulmonary metastases from primary colorectal cancers $(n=6 / 6)$. Heat map analyses of $\Delta C t$ values of the metastasis-related genes identified a 17-gene tropism signature that was sufficient not only to distinguish liver and the lung metastases, but also reconstituted the clustering of primary
\end{abstract}

Correspondence to: Dr Jhingook Kim, Department of Thoracic Surgery, Samsung Medical Center, Sungkyunkwan University, School of Medicine, 50 Ilwon-Dong, Kangnam-Ku, Seoul 135-710, Republic of Korea

E-mail: jkimsmc@skku.edu

Dr Ho-Kyung Chun, Department of Surgery, Samsung Medical Center, Sungkyunkwan University, School of Medicine, 50 IlwonDong, Kangnam-Ku, Seoul 135-710, Republic of Korea

E-mail: hkchun@skku.edu

*Contributed equally

Key words: colorectal cancer, liver, lung, metastasis, tropism, colonization tumors with the hepatic metastases $(n=17 / 18)$. In this pilot experiment, pulmonary metastases were significantly diverged from hepatic metastases that were indistinguishable from primary colorectal cancers. Further genomic and clinical studies are in progress to evaluate the potential of the tropism signature as a therapeutic target to inhibit the colonization of metastatic colorectal cancers.

\section{Introduction}

Metastasis proceeds through a multi-step process that can be divided into dissemination and colonization stages (1-3). Dissemination frequently occurs at early stages where disseminated tumor cells (DTCs) are detected in the peripheral blood or in the bone marrow (4-9). This is further supported by a high recurrence rate in certain types of cancers (10-12), providing a rationale for systemic adjuvant chemotherapy $(13,14)$. In contrast, colonization mechanisms are later activated at the metastatic site $(15,16)$, frequently after several years of dormancy (9). Colonization mechanisms can be acquired de novo in the primary tumors, and these clones might be selected, evolved and expanded at the metastatic sites (17). Another possibility is that these characteristics do not exist in the primary tumors but are induced by interaction with the metastatic microenvironments $(18,19)$, as supported by altered drug responsiveness when co-cultured with stromal cells (20-22) or when grafted to different organs (23). Regardless of when the colonization mechanisms evolve, they are activated in a metastatic site-specific manner. Recently, we conducted a longitudinal comparison during colon-to-lung metastasis to identify the molecular mechanisms of colonization at the gene expression level (24). In the present study, we performed similar expression analyses with additional genes and samples including colon-to-liver metastases and compared them with those from colon-to-lung metastases.

The two most frequent target organs of metastatic colorectal cancers (mCRCs) are the liver and the lung $(25,26)$, but the underlying metastatic tropism and the mechanisms determining which organ to metastasize remain controversial. In Paget's seed and soil hypothesis, metastatic clones are selected 
for their ability to grow in distant microenvironments (27-30), as supported by punctuated parallel evolution $(15,16,31)$. On the other hand, clinicians frequently observe microscopic portal vein thrombi trapped in the microvasculature of the liver, suggesting the importance of the anatomy of the blood flow for hepatic tropism $(25,32,33)$. The former hypothesis suggests that the newly activated colonization mechanism in the distant microenvironment is the key element during metastasis, whereas the latter suggests that the dissemination route plays a key role. Gene expression analyses of over 200 genes with known functions in metastasis, chemokine signals and angiogenesis might be sufficient to reveal the activated pathways. Nonetheless, relevant clinical samples are difficult to obtain because of the rarity of metastasectomy and decreasing integrity of the samples during the latent years. Fortunately, current trends toward more aggressive metastasectomy (34) may generate more clinical samples in the future.

The liver and the lung have different tissue structures, resident cell types $(35,36)$ and growth stimulants that are released. For example, IGF1, IGF2, HGF, MST (HGF-like) activator and RARRES2 are expressed at significantly higher levels in the liver, whereas GDF10, CSH1, BTG2, LTBP2, VWF, CSF3, IL6, FIGF, HBEGF, endothelin1, LTF, WIFP2 and VIM are higher in the lung (37). Hepatectomy has been reported to stimulate not only regeneration of the liver, but also the growth of colorectal cancers in animal experiments (38), indicating that wound healing signals in the liver might be conducive to mCRC cell growth. Indeed, HGF and its receptor MET were identified as prognostic markers for hepatic metastasis in CRCs $(39,40)$. In lung cancers, the prediction of metastasis with primary tumor gene expression signatures has resulted in limited success (41), raising the possibility that the metastatic potential of lung cancer may not be predetermined in the primary tumor. Taken together, it is likely that different colonization mechanisms are activated in different microenvironments, and here we provide molecular evidence suggesting that multiple distinct colonization mechanisms are activated in the lung in contrast to the liver that requires little change to be colonized by colorectal cancers.

\section{Materials and methods}

Patient and sample selection. Twelve pairs of primary colon tumor samples and matched hepatic or pulmonary metastases were collected from 12 patients (Table I) by surgical resection conducted at Samsung Medical Center between 2001 and 2008. The institutional review board approved the study protocol and prior consent was obtained from all patients. A clinical coordinator reviewed all medical records. Tumor specimens were stained with hematoxylin and eosin $(\mathrm{H} \& \mathrm{E})$ and examined by a pathologist to remove necrotic tissue and/or intervening stromal tissues and were classified according to the World Health Organization histopathological criteria. After necrotic regions were removed and the tumor masses were snap-frozen and stored in liquid nitrogen. Specimens were examined by pathologists to select the specimens with $>90 \%$ cancer cell content for RNA extraction and gene expression analyses.

Gene expression profiling using the $R T^{2}$ Profiler $^{T M} P C R$ array. To extract total-RNA from the tumor specimens, the NucleoSpin RNA kit was used following the manufacturer's protocol. Only RNA samples with a RIN value $>7.0$ were used as templates for cDNA synthesis using the $\mathrm{RT}^{2}$ First Strand kit (Cat. no. C-03; SABiosciences). Human tumor metastasis (PAHS-028), chemokines (PAHS-022) and angiogenesis (PAHS-024) finder $\mathrm{RT}^{2}$ profiler PCR arrays and $\mathrm{RT}^{2} \mathrm{SYBR}-$ Green/Rox PCR Master mix (APMM012C and PA-012-24; SABiosciences) were used to quantitatively analyze the gene expression levels of 84 metastasis genes (APC, BRMSI, CCL7, CD44, CDH1, CDH11, CDH6, CDKN2A, CHD4, COL4A2, CST7, CTBP1, CTNNA1, CTSK, CTSL1, CXCL12, CXCR4, DENR, EPHB2, ETV4, EWSR1, FAT, FGFR4, FLT4, FN1,

Table I. Summary of clinical information.

\begin{tabular}{|c|c|c|c|c|c|c|c|}
\hline \multirow[b]{2}{*}{ Case } & \multirow[b]{2}{*}{ Gender } & \multirow[b]{2}{*}{ Age (years) } & \multicolumn{3}{|c|}{ Primary tumor } & \multicolumn{2}{|c|}{ Metastatic tumor } \\
\hline & & & Size $(\mathrm{cm})$ & pStage $^{a}$ & Primary site & $\operatorname{Size}^{\mathrm{b}}(\mathrm{cm})$ & Metastatic site \\
\hline 1 & M & 72 & 7.7 & III & S colon & 3.5 & Liver \\
\hline 2 & M & 47 & 10.0 & III & Lower rectum & 4.3 & Liver \\
\hline 3 & $\mathrm{~F}$ & 66 & 2.7 & III & Lower rectum & 2.7 & Liver \\
\hline 4 & F & 69 & 6.0 & III & Colon & 1.7 & Liver \\
\hline 5 & $\mathrm{~F}$ & 70 & 4.8 & III & Colon & 2.7 & Liver \\
\hline 6 & M & 59 & 9.0 & III & Colon & 4.0 & Liver \\
\hline 7 & $\mathrm{~F}$ & 36 & 4.8 & III & Lower rectum & 2.0 & Lung \\
\hline 8 & $\mathrm{~F}$ & 49 & 4.4 & III & Lower rectum & 3.4 & Lung \\
\hline 9 & $\mathrm{~F}$ & 57 & 5.2 & III & Upper rectum & 3.0 & Lung \\
\hline 10 & M & 65 & 3.5 & I & Lower rectum & 3.2 & Lung \\
\hline 11 & M & 70 & 3.5 & II & Upper rectum & 1.8 & Lung \\
\hline 12 & $\mathrm{~F}$ & 53 & 5.2 & III & S-colon & 1.0 & Lung \\
\hline
\end{tabular}

${ }^{a}$ pStage at the time of the primary surgery; ${ }^{b}$ diameter of the largest tumor nodule. 


\section{A 84 Metastasis genes}

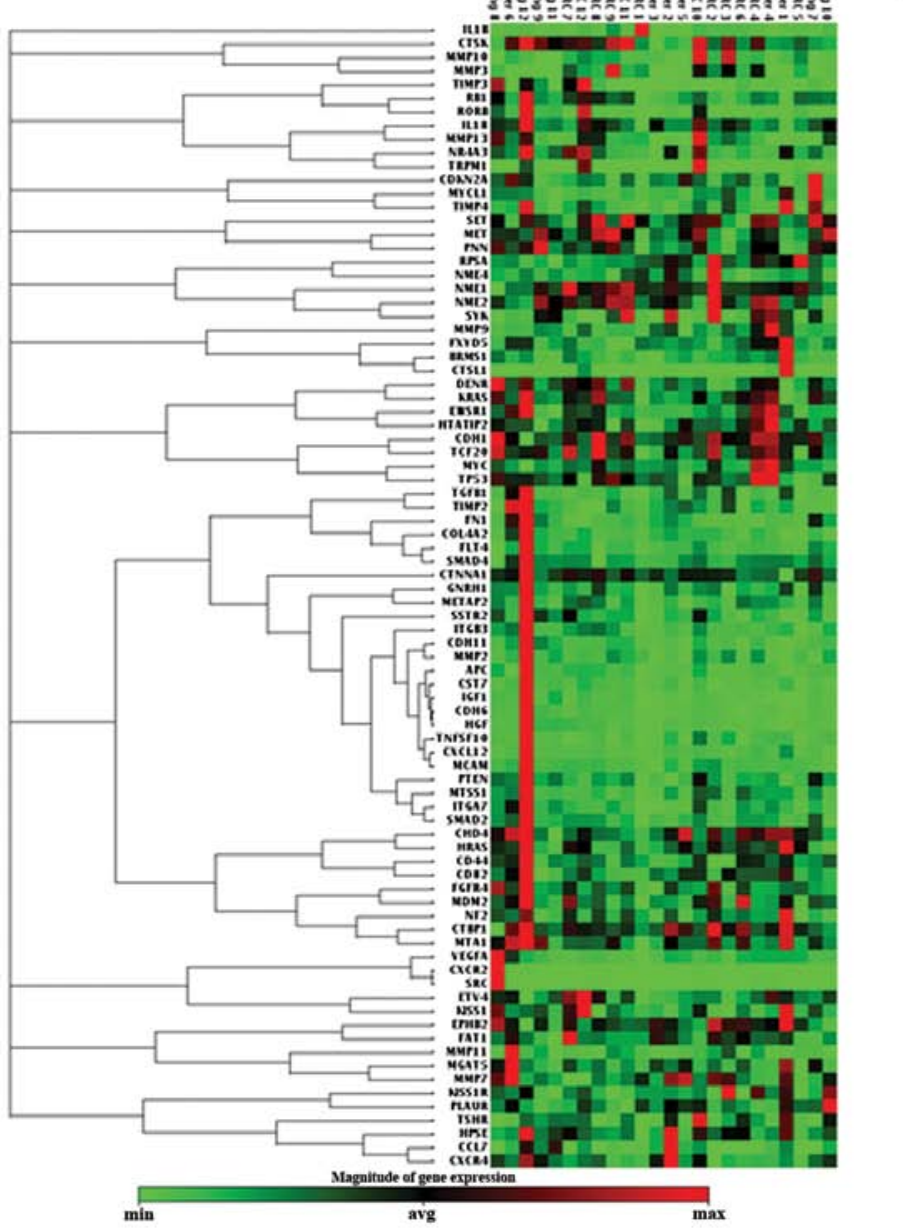

A-1

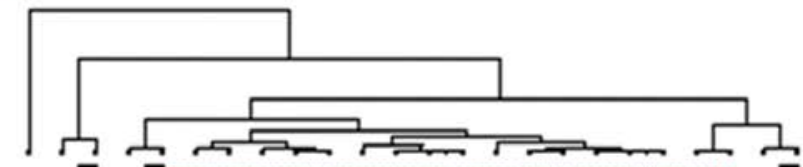

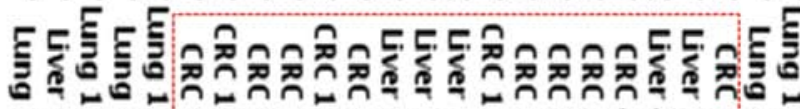
の の

B-1

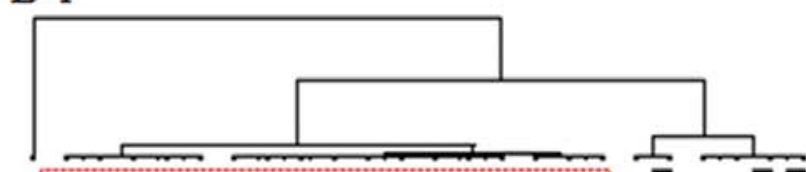

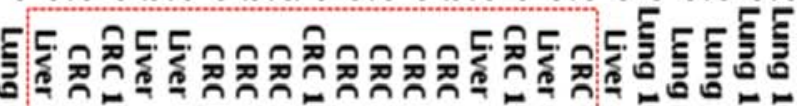
$\infty$ 는

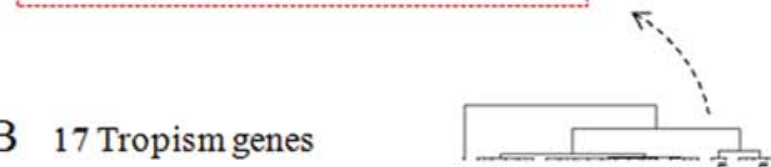

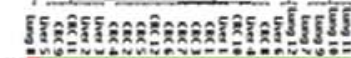

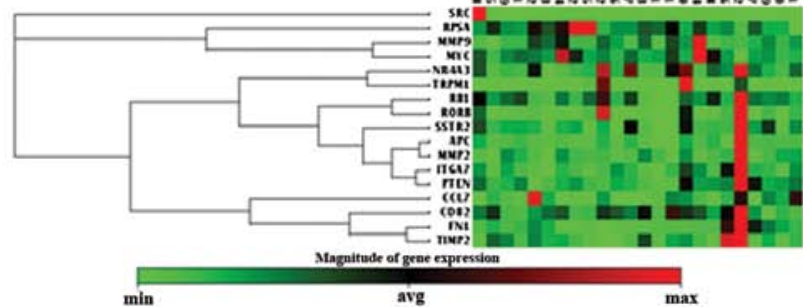

Figure 1. Metastatic tropism of CRCs: divergence between pulmonary and hepatic metastases. Unbiased hierarchical clustering with $\mathrm{Ct}$ values from 24 tumor samples. (A) All 84 genes from the metastasis array were used to generate the clustergram. All CRCs (primary tumors) formed one cluster with hepatic metastases. Lung groups 7-12 (pulmonary metastases) diverged from one another and from the other tumors. (B) The 17-tropism signature reconstructed the pulmonary divergence. In the order of most remote: Lung 8, followed by Lung 12 and Liver 6, and Lungs 7, 9, 10 and 11 . Similarity between the hepatic and primary CRCs was also reconstructed. Stratification within this group seems to depend on minute and insignificant differences. CRC 1-6, primary colorectal cancers matching Livers 1-6 (hepatic metastases). CRC 7-12, primary colorectal cancers matching Lungs 7-12 (pulmonary metastases). Liver 6 was an exceptional case to cluster with Lung 12. Red box, clusters formed by CRCs and Liver.

FXYD5, GNRH1, KISSIR, HGF, HPSE, HRAS, HTATIP2, IGF1, IL18, ILIB, IL8RB, ITGA7, ITGB3, CD82, KISS1, KRAS, RPSA, MCAM, MDM2, MET, METAP2, MGAT5, MMP10, MMP11, MMP13, MMP2, MMP3, MMP7, MMP9, MTA1, MTSS1, MYC, MYCL1, NF2, NME1, NME2, NME4, NR4A3, PLAUR, PNN, PTEN, RB1, RORB, SET, SMAD2, SMAD4, SRC, SSTR2, SYK, TCF20, TGFB1, TIMP2, TIMP3, TIMP4, TNFSF10, TP53, TRPM1, TSHR, VEGFA), 84 chemokines and receptor genes (CCL1, CCL11, CCL13, CCL15, CCL16, CCL17, CCL18, CCL19, CCL2, CCL3, CCL4, CCL5, CCL7, CCL8, CXCL1, CXCL10, CXCL11, CXCL12, CXCL13, CXCL2, CXCL3, CXCL5, CXCL6, CXCL9, CCR1, CCR2, CCR3, CCR4, CCR5, CCR6, CCR7, CCR8, CCR10, CCRL1, CCRL2, CXCR3, CXCR4, CXCR6, CYFIP2, APLNR, BDNF, C5, C5AR1 GPR77, CCBP2, CKLF, CMTM1, CMTM2, CMTM3, CMTM4, CMKLR1, CSF3, CX3CL1, CX3CR1, TYMP, GDF5, GPR31, HCAR1, HIF1A, IL13, IL16, IL18, IL1A, IL4, IL8, CXCR1, LTB4R, MMP2, MMP7, MYD88, NFKB1, AIMP1, SDF2, SLIT2, TCP10, TLR2, TLR4, TNF,
TNFRSF1A, TNFSF14, TREM1, VHL, XCL1, XCR1), 84 angiogenesis genes (ANGPT1, ANGPT2, ANPEP, TYMP, EREG, FGF1, FGF2, FIGF, FLT1, JAG1, KDR, LAMA5, NRP1, NRP2, PGF, PLXDC1, STAB1, VEGFA, VEGFC, ANGPTL3, BAII, COL4A3, IL8, LAMA5, NRP1, NRP2, STAB1, ANGPTL4, PECAM1, PF4, PROK2, SERPINF1, TNFAIP2, HAND2, SPHK1, CCL11, CCL2, CXCL1, CXCL10, CXCL3, CXCL5, CXCL6, CXCL9, IFNA1, IFNB1, IFNG, ILIB, IL6, MDK, TNF, SIPR1, EFNA1, EFNA3, EFNB2, EGF, EPHB4, FGFR3, HGF, IGF1, ITGB3, PDGFA, TEK, TGFA, TGFB1, TGFB2, TGFBR1, CCL11, CDH5, COL18A1, SIPR1, ENG, ITGAV, ITGB3, THBS1, THBS2, LECT1, LEP, MMP2, MMP9, PLAU, PLG, TIMP1, TIMP2, TIMP3, AKT1, HIF1A, HPSE, IDI, ID3, NOTCH4, PTGS1). Five housekeeping genes (B2M, HPRT1, RPL13A, GAPDH, ACTB) and MMP2 were triplicated, and twenty-seven genes were $\mathrm{d}(C C L 7$, CXCL12, CXCR4, HGF, IGF1, IL18, IL1B, ITGB3, MMP2, MMP3, MMP9, TGFB1, TIMP2, TIMP3, VEGFA, TYMP, CCL11, CCL2, CXCL1, CXCL10, CXCL3, CXCL5, CXCL6, 
Table II. mCRC tropism signature.

\begin{tabular}{lcc}
\hline No. & Gene symbol & Fold change \\
\hline 1 & APC & 6.23 \\
2 & MMP2 & 4.23 \\
3 & RORB & 4.01 \\
4 & SSTR2 & 3.56 \\
5 & ITGA7 & 2.84 \\
6 & RB1 & 2.58 \\
7 & PTEN & 1.92 \\
8 & TRPM1 & 1.92 \\
9 & NR4A3 & 1.83 \\
10 & SRC & 1.53 \\
11 & FN1 & 1.52 \\
12 & TIMP2 & -1.56 \\
13 & MMP9 & -1.59 \\
14 & CD82 & -1.71 \\
15 & RPSA & -1.72 \\
16 & CCL7 & -1.93 \\
17 & MYC & -2.02 \\
\hline
\end{tabular}

CXCL9, TIMP2, TIMP3, HIF1A, HPSE). Negative controls for contamination were included in the 96-well PCR arrays. RT-PCR was conducted using an ABI 7300 Real Time PCR system (Applied Biosystems).

Data analysis. $\mathrm{Ct}$ values $>35$ were considered to indicate either reaction failure or absence of expression and were excluded from the data analyses. The amount of sample loaded per well was estimated and normalized according to the expression levels of six housekeeping genes. Differential gene expression was estimated as follows: $\Delta \mathrm{Ct}=\mathrm{Ct}_{\text {(lung metastases) }}-\mathrm{Ct}_{\text {(colon primary) }}$ and fold change $=2^{(-\Delta \mathrm{Ct})}$. Quantitative analyses including hierarchical clustering, heat map analyses and volcano plots of the array data for the primary colon and metastatic tumors were conducted using Web-based PCR array data analysis software (http://www.sabiosciences.com). The metastatic signature tropism was selected by progressive removal of redundant genes from the metastasis arrays for the maintenance of the original clustering pattern. Removal of any single gene from the 17-gene signature disrupted the original clustering pattern.

\section{Results}

Quantitative gene expression. The gene expression level of 224 genes was determined quantitatively for a total of 24 CRC tumors, 12 pairs of primary-to-metastatic tumors including six colon-to-liver (no. 1-6) pairs and six colon-to-lung (no. 7-12) pairs. $\mathrm{Ct}$ values were normalized against the deviation from the median of the five housekeeping genes. The $\mathrm{Ct}$ values for 27 duplicate or 6 triplicate genes were highly reproducible for assayable genes (41) with activated expression. For those genes with expression at basal levels or lower, $\mathrm{Ct}$ values $>35$ were collectively considered as no expression and were excluded from further analysis. Among the assayable genes, the average correlation between duplicates was 0.92 .
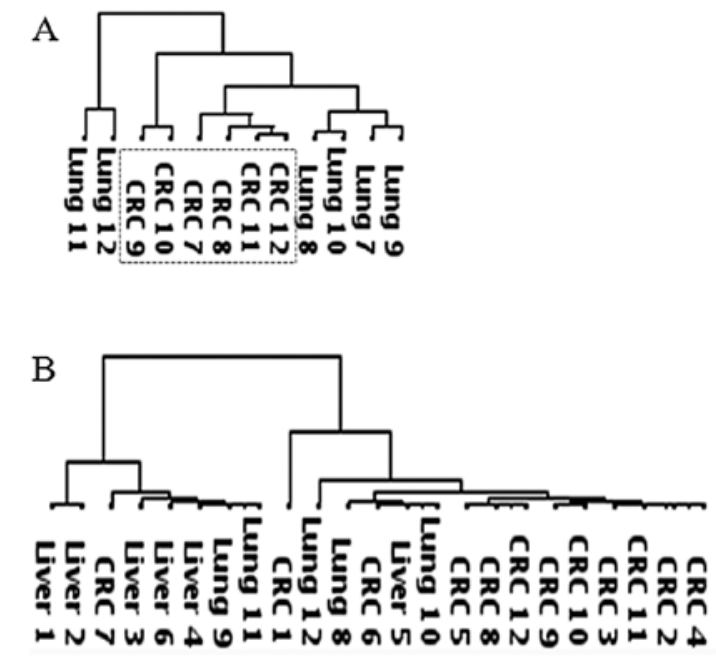

Figure 2. Hierarchical clustering of chemokine- and angiogenesis-related genes. (A) Phylogenetic tree from clustergram of 84 chemokines and receptors in 6 cases with pulmonary metastases. Due to shortage of mRNA amount, data for hepatic cases were not generated. (B) Phylogenetic tree from clustergram of 84 angiogenesis genes did not stratify hepatic and pulmonary cases.

Metastatic tropism: hepatic vs. pulmonary CRC metastases of $C R C s$. To overview the relative similarities or differences among the samples, an unsupervised two-dimensional hierarchical clustering was conducted with the $\mathrm{Ct}$ values. In the metastasis array data, all hepatic metastases formed closely related clusters with the primary CRCs with one exception of Liver 6 (Fig. 1A). The clinical information was examined to determine the possibility of Liver 6 being a secondary metastasis from pulmonary metastasis, but no pulmonary metastasis was detected in that patient. Among the six cases of hepatic metastases, only one original tumor pair was clustered together (CRC4 and Liver 4) and the rest clustered with a tumor from a different patient, indicating that the similarities between the hepatic metastases and the primary CRCs were greater than the person-to-person diversities in the tested samples. In contrast to the hepatic metastases, the pulmonary metastases were highly diversified from one another and from the primary colorectal tumors (Fig. 1A). To identify potential markers for tropism, heat map analyses were performed with the $\Delta \mathrm{Ct}$ values between the hepatic and pulmonary metastases (data not shown). Forty-six genes were differentially regulated $(>1.5$-fold) when the hepatic and pulmonary metastases were compared. From the listed genes, those with similar expression patterns were removed to finalize the 17-gene tropism signature (Table II). To confirm that the tropism signature was sufficient to distinguish the hepatic from the pulmonary metastases, all of the 24 tumor samples were analyzed again by unsupervised hierarchical clustering (Fig. 1B). The 17-gene signature not only distinguished the liver metastases from the lung metastases, but also clustered primary CRCs with the hepatic metastases, reconstituting the clustergram of 84 metastasis genes. Chemokine array data were obtained only for the pulmonary metastases and their primary CRCs due to a shortage of RNA samples. Hierarchical clustering of the chemokine array data also showed that the pulmonary metastases were diversified from the primary CRCs (Fig. 2A). Tropism genes could not be identified from the hepatic metastases due to the lack of chemokine array 

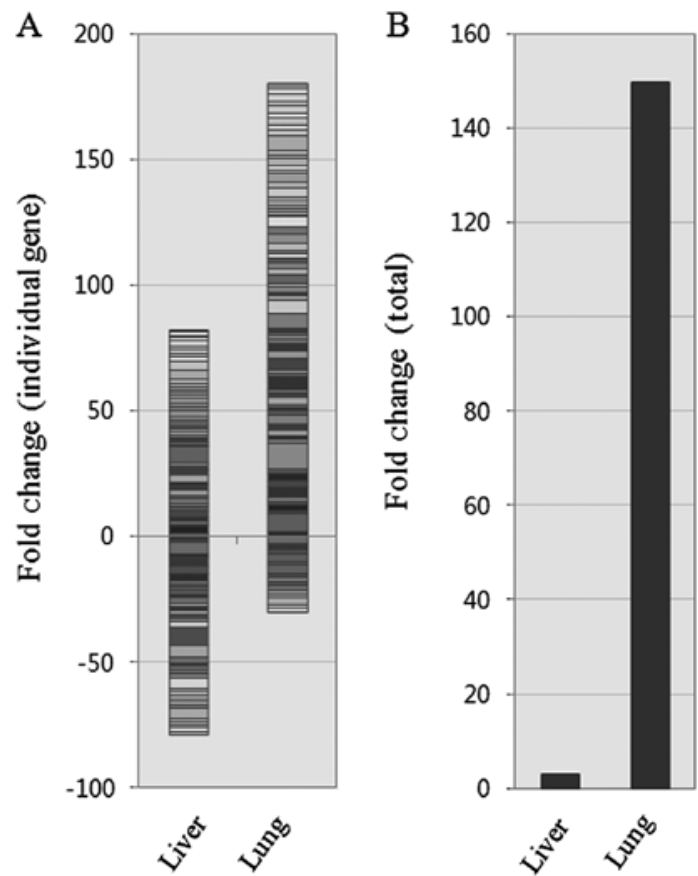

Figure 3. Overall activation of angiogenesis-related genes in pulmonary metastases. (A) All 84 angiogenesis array data sets were used. The majority of genes were upregulated in the pulmonary metastases, in contrast to hepatic metastases with balanced up- and downregulation. (B) Sum of fold-change values and visualization of the overall up- or downregulation. Hepatic metastases showed little upregulation compared with CRCs, but the pulmonary metastases showed significant (150-fold) overall upregulation. Relative amounts of RNA samples were determined and normalized by the expression levels of $B 2 M, G A P D H, A C T B$ and RPL13A, fold change was calculated by the $2^{-\Delta \mathrm{Ct}}$ method and the data were plotted. No significant array-to-array difference was detected in the quality and quantity of mRNA.

data. Nonetheless, expression profiling of the 17-tropism genes was sufficient to distinguish the hepatic and pulmonary CRC metastases and to show similarity between the hepatic and primary CRCs in the tested samples.

Overall activation of angiogenic pathways in pulmonary metastases. To examine the possible role of angiogenic pathways in late steps of colonization, supporting the growth of micrometastases into a detectible size, angiogenesis array data were obtained for all 24 tumor samples. Unlike the metastasis array and the chemokine array data, hierarchical clustering of the angiogenesis array data did not group the pulmonary metastases and the hepatic metastases into separate clusters (Fig. 2B). However, when overall gene expression was examined between these groups, the total fold change was significantly upregulated ( 150-fold) during colon-to-lung metastasis compared to colon-to-liver metastasis (Fig. 3). These data indicate that the diversity of angiogenic pathways is activated in the tested samples of pulmonary metastases.

\section{Discussion}

In metastatic cases, primary tumor surgical specimens are already equipped with dissemination mechanisms; therefore, longitudinal comparison with actively colonizing metastatic tumors would unveil the colonization mechanisms of the micrometastases. In this study we compared six pairs of colon- to-lung metastases with colon-to-liver metastases to understand how colonization mechanisms differ in the liver and the lung, the two most frequent target organs for metastatic CRCs.

Unsupervised hierarchical clustering of the metastasis array data revealed that the pulmonary and hepatic metastases were intrinsically different, with the exception of Liver 6 that clustered with Lung 12. All 84 metastasis array genes were not required for distinction between the two groups. Among the differentially expressed genes, a minimal 17-gene tropism signature was determined and was shown to separately cluster these two sample groups (Fig. 1). Interestingly, clustergrams with either 84 metastasis genes or 17 tropism genes demonstrated that all primary CRCs were similar to the hepatic metastases. These data suggest that the divergence of pulmonary metastases was not an over-interpretation of technical artifacts, such as major stromal contamination or random/uncontrolled events that can occur in any collection of samples. In addition, significant divergence did not occur in the primary tumors. If divergence of metastatic potential is manifested in the primary tumors, gene expression profiles of the primary tumors should be able to predict recurrence, which is not the case in lung cancers (41). Lastly, the liver and colon might have such similar microenvironments that little change is needed for CRCs to cross-colonize between them, which is consistent with previous reports (42) and a classical view of passive transport to amiable microenvironments in the liver via the portal vein $(32,33)$. Although the number of samples tested was too small to exert a definitive conclusion, the divergence of pulmonary metastases from one another raises the possibility that there might be multiple distinct metastatic microenvironments in the lung.

Angiogenesis needs to be activated for both primary and metastatic tumors to grow into a detectible size, and consequently not specific to either metastatic colonization or tropism. Our data showed that the expression profiles of angiogenesis-related genes were not specific to the tumors formed in the liver, the lung or the colorectal microenvironments. Nonetheless, overall expression in the pulmonary metastases was $~ 150$-fold higher than those in the hepatic metastases and the primary CRCs (Fig. 3). This might be an adaptive change to the relatively sterile microenvironments of the lung compared with the strong regenerative potentials found in the liver and the digestive track.

In this study we examined differentially activated genes during colon-to-liver and colon-to-lung metastases to learn that pulmonary metastases were highly diversified in contrary to hepatic metastases that were indistinguishable from primary CRCs and identified a 17-gene tropism signature. Our data suggest that pulmonary metastases need different therapeutics from hepatic metastases and primary CRCs; however, further clinical studies with larger sample sizes are required to validate this conclusion.

\section{Acknowledgements}

This study was supported by grants from the Samsung Biomedical Research Institute (no. SBRI C-A6-411, C-A7-802), the National Research Foundation of Korea (NRF) funded by the Korean government (MEST) (no. R01-2006-000-11114-0, and NTX2091113) and the Seoul R\&BD Program (SS100010). 


\section{References}

1. Nguyen DX, Paula DB and Massagué J: Metastasis: from dissemination to organ-specific colonization. Nat Rev Cancer 9: 274-284, 2009

2. Fidler IJ: Critical factors in the biology of human cancer metastasis: twenty-eighth G.H.A. Clowes memorial award lecture. Cancer Res 50: 6130-6138, 1990.

3. Talmadge JE and Fidler IJ: AACR centennial series: the biology of cancer metastasis: historical perspective. Cancer Res 70 $5649-5669,2010$.

4. Hanahan D and Weinberg RA: Hallmarks of cancer: the next generation. Cell 144: 646-674, 2011.

5. Pantel K and Brakenhoff RH: Dissecting the metastatic cascade. Nat Rev Cancer 4: 448-456, 2004.

6. Braun S, Vogl FD, Naume B, et al: A pooled analysis of bone marrow micrometastasis in breast cancer. N Engl J Med 353: 793-802, 2005.

7. Hüsemann Y, Geigl JB, Schubert F, et al: Systemic spread is an early step in breast cancer. Cancer Cell 13: 58-68, 2008.

8. Lin H, Balic M, Zheng S, Datar R and Cote RJ: Disseminated and circulating tumor cells: Role in effective cancer management. Crit Rev Oncol Hematol 77: 1-11, 2011.

9. Flatmark K, Borgen E, Nesland JM, et al: Disseminated tumor cells as a prognostic biomarker in colorectal cancer. $\mathrm{Br} \mathrm{J}$ Cancer 104: 1434-1439, 2011.

10. Sugimura H, Nichols FC, Yang P, et al: Survival after recurrent non small-cell lung cancer after complete pulmonary resection. Ann Thorac Surg 83: 409-418, 2007.

11. Feld R, Rubinstein LV and Weisenberger TH: Sites of recurrence in resected stage I non-small-cell lung cancer: a guide for future studies. J Clin Oncol 2: 1352-1358, 1984.

12. Gutt R, Liauw SL and Weichselbaum RR: Adjuvant radiotherapy for resected pancreatic cancer: a lack of benefit or a lack of adequate trials? Nat Clin Pract Gastroenterol Hepatol 6: 38-46, 2009.

13. Schabel FM Jr: Rationale for adjuvant chemotherapy. Cancer 39: 2875-2882, 1977.

14. Visbal AL, Leighl NB, Feld R and Shepherd FA: Adjuvant chemotherapy for early-stage non-small cell lung cancer. Chest 128: 2933-2943, 2005

15. Navin N, Kendall J, Troge J, et al: Tumour evolution inferred by single-cell sequencing. Nature 472: 90-94, 2011.

16. Campbell PJ, Yachida S, Mudie LJ, et al: The patterns and dynamics of genomic instability in metastatic pancreatic cancer. Nature 467: 1109-1113, 2010.

17. Greaves $M$ and Maley CC: Clonal evolution in cancer. Nature 481: 306-313, 2012.

18. Malanchi I, Santamaria-Martinez A, Susanto E, et al: Interactions between cancer stem cells and their niche govern metastatic colonization. Nature 481: 85-89, 2011.

19. Navab R, Strumpf D, Bandarchi B, et al: Prognostic gene-expression signature of carcinoma-associated fibroblasts in non-smal cell lung cancer. Proc Natl Acad Sci USA 108: 7160-7165, 2011.

20. Mink SR, Vashistha S, Zhang W, et al: Cancer-associated fibroblasts derived from EGFR-TKI-resistant tumors reverse EGFR pathway inhibition by EGFR-TKIs. Mol Cancer Res 8: 809-820, 2010.

21. Wang W, Li Q, Yamada T, et al: Crosstalk to stromal fibroblasts induces resistance of lung cancer to epidermal growth factor receptor tyrosine kinase inhibitors. Clin Cancer Res 15: 6630-6638, 2009.

22. Serebriiskii I, Castelló-Cros R, Lamb A, Golemis EA and Cukierman E: Fibroblast-derived 3D matrix differentially regulates the growth and drug-responsiveness of human cancer cells. Matrix Biol 27: 573-585, 2008
23. Wilmanns C, Fan D, O'Brian CA, Bucana CD, and Fidler IJ: Orthotopic and ectopic organ environments differentially influence the sensitivity of murine colon carcinoma cells to doxorubicin and 5-fluorouracil. Int J Cancer 52: 98-104, 1992.

24. Kim S-H, Choi S, Cho YB, et al: Differential gene expression during colon-to-lung metastasis. Oncol Rep 25: 629-36, 2011.

25. Gilson N, Honoré O, Detry O, et al: Surgical management of hepatic metastases of colorectal origin. Acta Gastroenterol Belg 72: 321-326, 2009

26. Kjeldsen BJ, Kronborg O, Fenger $\mathrm{C}$ and Jorgensen OD: The pattern of recurrent colorectal cancer in a prospective randomized study and the characteristics of diagnostic tests. Int $\mathrm{J}$ Colorectal Dis $12: 329-334,1997$.

27. Fidler IJ and Kripke ML: Genomic analysis of primary tumors does not address the prevalence of metastatic cells in the population. Nat Genet 34: 23, 2003.

28. Jones S, Chen WD, Parmigiani G, et al: Comparative lesion sequencing provides insights into tumor evolution. Proc Natl Acad Sci USA 105: 4283-4288, 2008.

29. Fidler IJ: The pathogenesis of cancer metastasis: the 'seed and soil' hypothesis revisited. Nat Rev Cancer 3: 453-458, 2003.

30. Psaila B and Lyden D: The metastatic niche: adapting the foreign soil. Nat Rev Cancer 9: 285-293, 2009.

31. Klein CA: Parallel progression of primary tumours and metastases. Nat Rev Cancer 9: 302-312, 2009.

32. Gupta GP, and Massague J: Cancer metastasis: building a framework. Cell 127: 679-695, 2006.

33. Yamamoto J, Sugihara K, Kosuge T, et al: Pathologic support for limited hepatectomy in the treatment of liver metastasis from colorectal cancer. Ann Surg 221: 74-78, 1995.

34. Park JS, Kim HK, Choi YS, et al: Outcomes after repeated resection for recurrent pulmonary metastases from colorectal cancer. Ann Oncol 21: 1285-1289, 2010.

35. Suriawinata AA and Thung NS: Liver. In: Histology for Pathologists. Mills SE (ed). 3rd edition. Lippincott Williams \& Wilkins, pp685-703, 2007.

36. Colby TV, Leslie KO and Yousem SA: Lung. In: Histology for Pathologists. Mills SE (ed). 3rd edition. Lippincott Williams \& Wilkins, pp473-504, 2007.

37. Stelzer G, Inger A, Olender T, et al: GeneDecks: paralog hunting and gene-set distillation with GeneCards annotation. OMICS 13: 477-487, 2009.

38. Fidler IJ: Critical factors in the biology of human cancer metastasis: twenty-eighth G.H.A. Clowes memorial award lecture. Cancer Res 50: 6130-8, 1990.

39. Kopitz C, Gerg M, Bandapalli OR, et al: Tissue inhibitor of metalloproteinases-1 promotes liver metastasis by induction of hepatocyte growth factor signaling. Cancer Res 67: 8615-8623, 2007.

40. Stein U, Walther W, Arlt F, et al: MACC1, a newly identified key regulator of HGF-MET signaling, predicts colon cancer metastasis. Nat Med 15: 59-67, 2009.

41. Lee ES, Son DS, Kim S-H, et al: Prediction of recurrence-free survival in postoperative non-small cell lung cancer patients by using an integrated model of clinical information and gene expression. Clin Cancer Res 14: 7397-7404, 2008.

42. Koh KH, Rhee H, Kang HJ, et al: Differential gene expression profiles of metastases in paired primary and metastatic colorectal carcinomas. Oncology 75: 92-101, 2008. 